\title{
A Note on Amino Acids Essential for Growth of Young Carp*1
}

\author{
Takeshi NoSE*2, Shigeru ARAI*2, Dong-Liang LEE*3, \\ and Yoshiro HashIMOTO*3
}

(Received May, 31, 1974)

\begin{abstract}
Young carp, Cyprinus carpio, were found to grow to some extent on an amino acid test diet neutralized with $\mathrm{NaOH}$, but only a little on a diet not neutralized. Thirty five fish were grouped in each aquarium and fed ad libitum the test diet adjusted to $\mathrm{pH} 6.5 \sim 6.7$ for a period of 6 weeks. The fish fed diets deficient in each of alanine, aspartic acid, cystine, glutamic acid, glycine, proline, tyrosine and serine grew as well as those fed the complete diet. The fish fed diets deficient in each of arginine, histidine, isoleucine, leucine, lysine, methionine, phenylalanine, threonine, tryptophan and valine failed to grow until the deleted amino acid was returned to the ration. It was thus postulated that carp require the same ten kinds of amino acid reported to be essential for other fishes.
\end{abstract}

Several species of fish, such as chinook salmon, sockeye salmon, rainbow trout, channel catfish and eel ${ }^{1-5)}$ have been shown to require the same ten amino acids for the normal growth with the diet in which protein was replaced by crystalline L-amino acids. It was however reported by AoE et al. ${ }^{\text {3) }}$ that the amino acid test diet could not sustain the growth of young carp Cyprinus carpio, even when modified in various ways. The amino acids essential for carp have been thus still unknown.

In the light of the fact that the amino acid test diet is one of the important tools for the study of protein nutrition, we attempted to establish the amino acid test diet for carp. After repeated preliminary experiments, we succeeded in sustaining the growth of young carp to some extent with an amino acid test diet, and this allowed us to postulate that carp require the same ten kinds of amino acid as the above-mentioned fishes.

The present paper deals with these results.

\section{Materials and Methods}

Test diets All the amino acids used were in L-form. Arginine, histidine and lysine were in the hydrochloride form and others in the free form.

The composition of the basal diet is shown in Table 1 and almost the same as those used in our previous work ${ }^{5}$ on eels. The amino acid mixture was prepared after the formulation developed by HALVER for chinook salmon" , but its level in the diet was fixed

*1 Freshwater Fisheries Research Laboratory, Contribution No. 337.

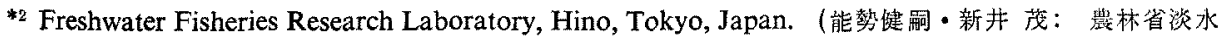
区水應研究所)

*3 Laboratory of Marine Biochemistry, Faculty of Agriculture, The University of Tokyo, Bunkyo-ku, Tokyo, Japan. (李楝樑・橋本芳郎: 東京大学農学部水侜化学研究室) 
Table 1. Composition of the basal diet

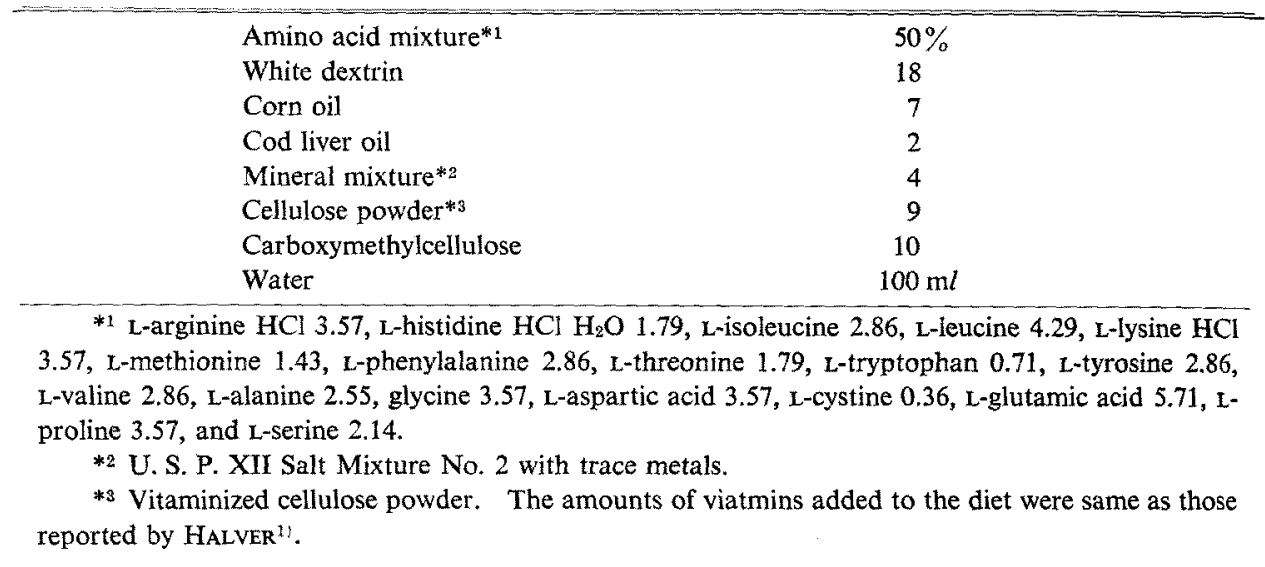

at $50 \%$. In the deficient diets, the amino acid deleted from the basal diet was replaced by cellulose powder. The test diets were prepared as paste and preserved in a freezer at $-20^{\circ} \mathrm{C}$ until used.

To adjust the $\mathrm{pH}$ value of diet, a necessary quantity of $6 \mathrm{~N} \mathrm{NaOH}$ was added to the water ingredient before mixing with dry diet mixture. The $\mathrm{pH}$ value was estimated with a glass-electrode $\mathrm{pH}$ meter on the supernatant obtained by homogenizing a $5 \mathrm{~g}$ portion of diet with $50 \mathrm{~m} l$ of distilled water.

Experimental fish and feeding method Young carp weighing about $2 \mathrm{~g}$ were obtained from Saitama Prefectural Fisheries Station in June 1972, and kept in tanks on a commercial diet. The fish were sorted two weeks before the start of experiment and kept on the basal diet in aquariums $(20 \times 20 \times 50 \mathrm{~cm})$ made of polyvinyl chloride. To each aquarium well water adjusted to $25^{\circ} \mathrm{C}$ was supplied after aeration at a rate of $1.21 / \mathrm{min}$.

Thirty-five fish were grouped in each aquarium for each experimental diet. They were fed six times a day from 9:00 a.m. at an interval of $90 \mathrm{~min}$ except Sunday when they were fed three times. The pasty diet was extruded into tanks as moist fine noodle from a $50 \mathrm{~m} l$ nylon syringe without needle. The diet was fed so long as the fish actively fed it at each feeding time. Aquariums were cleaned with nylon brush every two days without removing fish. Each individual was weighed to the nearest $0.01 \mathrm{~g}$ after being anesthetized in a MS $222(1: 10,000)$ solution at intervals of 2 weeks.

\section{Results}

Effect of diet $\mathrm{pH}$ on growth of young carp In preliminary experiments, we noticed a sign that the $\mathrm{pH}$ value of amino acid test diet affects considerably the growth of carp. To ascertain this, diets were adjusted to different $\mathrm{pH}$ values with $6 \mathrm{~N} \mathrm{NaOH}$. The relation between $\mathrm{pH}$ values of diet and the quantity of $6 \mathrm{~N} \mathrm{NaOH}$ per $10 \mathrm{~g}$ of dry diet mixture is 
shown in Fig. 1. The diet shows $\mathrm{pH} 4.0$ before adjustment and requires a considerable quantity of $6 \mathrm{~N} \mathrm{NaOH}$ for neutralization. Young carp were then raised with the test diets of different $\mathrm{pH}$ values for 4 weeks. The percent body weight gain is given in Fig. 2. The fish fed actively the diet which had not been neutralized but grew only a little, the feed efficiency being as low as $6 \%$. On the other hand, fish receiving diets adjusted to over $\mathrm{pH} 5.0$ reveal far better growth than those receiving the diet of $\mathrm{pH} 4.0$. Effect of $\mathrm{pH}$ value over 5.0 on weight gain is insignificant.

Essential and nonessential amino acids for young carp Since carp were found to grow to some extent on the basal diet adjusted to $\mathrm{pH}$ over 5.0, essential and nonessential amino acids were examined by eliminating each amino acid from the basal diet adjusted to $\mathrm{pH}$ $6.5 \sim 6.7$. The results of the feeding experiment are shown in Fig. 3. The fish on diets

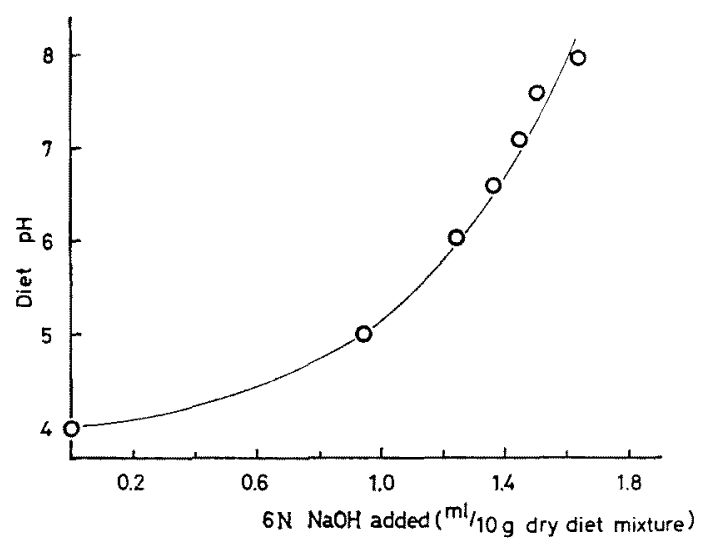

Fig. 1. The quantity of $6 \mathrm{~N} \mathrm{NaOH}$ and $\mathrm{pH}$ of test diet.

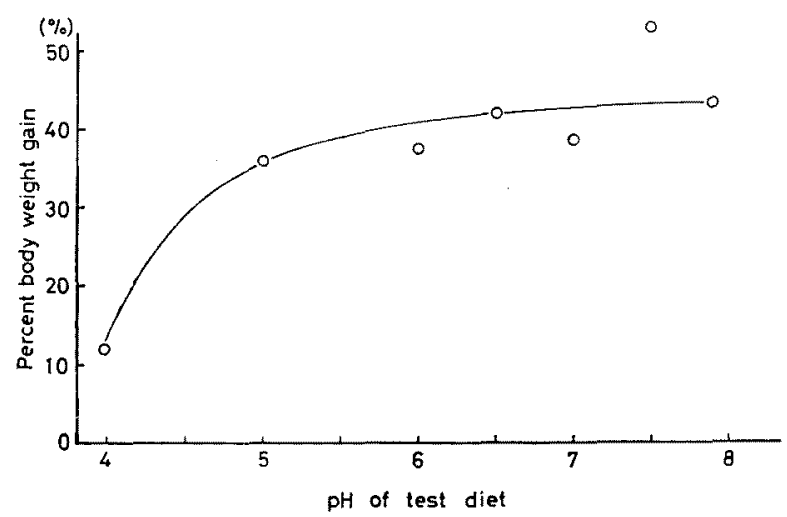

Fig. 2. Relation between pH of test diet and growth of carp. 
deficient in each of arginine, histidine, lysine, isoleucine, leucine, methionine, phenylalanine, threonine, tryptophan, and valine show retarded growth and remarkable difference in the average body weight from those on the basal diet at the end of 4 weeks, indicating that these amino acids are essential. On the other hand, the fish on diets deficient in each of alanine, glycine, aspartic acid, glutamic acid, tyrosine, cystine, proline and serine show a body weight gain similar to that of fish on the basal diet.

From the beginning of the 5 th week, recovery test was conducted with the groups showing retarded growth by replacing the deficient diets with the basal biet. In the recovery test, the fish show a substantial growth response to the basal diet as shown in Fig. 3.

In the course of feeding experiment, congestion of both caudal and pectoral fins was recognized on a small number of fish in almost all the essential amino acid-deficient groups and, in the latter half of feeding experiment, the symptom spread not only to some of the nonessential amino acid-deficient groups but also to the control groups. Fish in essential amino acid-deficient groups, except isoleucine- and lysine-deficient groups, showed some mortality in the period of recovery test, while those in nonessential amino acid-deficient groups no mortality. The highest mortality was $19 \%$ in both methionine- and tryptophandeficient groups, followed by valine- and histidine-deficient groups $(16 \%)$ and phenylalanine-deficient one $(13 \%)$ and others were less than $10 \%$. Besides, lordosis was observed in some of the fish in each groups except cystine-deficient one at end of the 4 th week. The
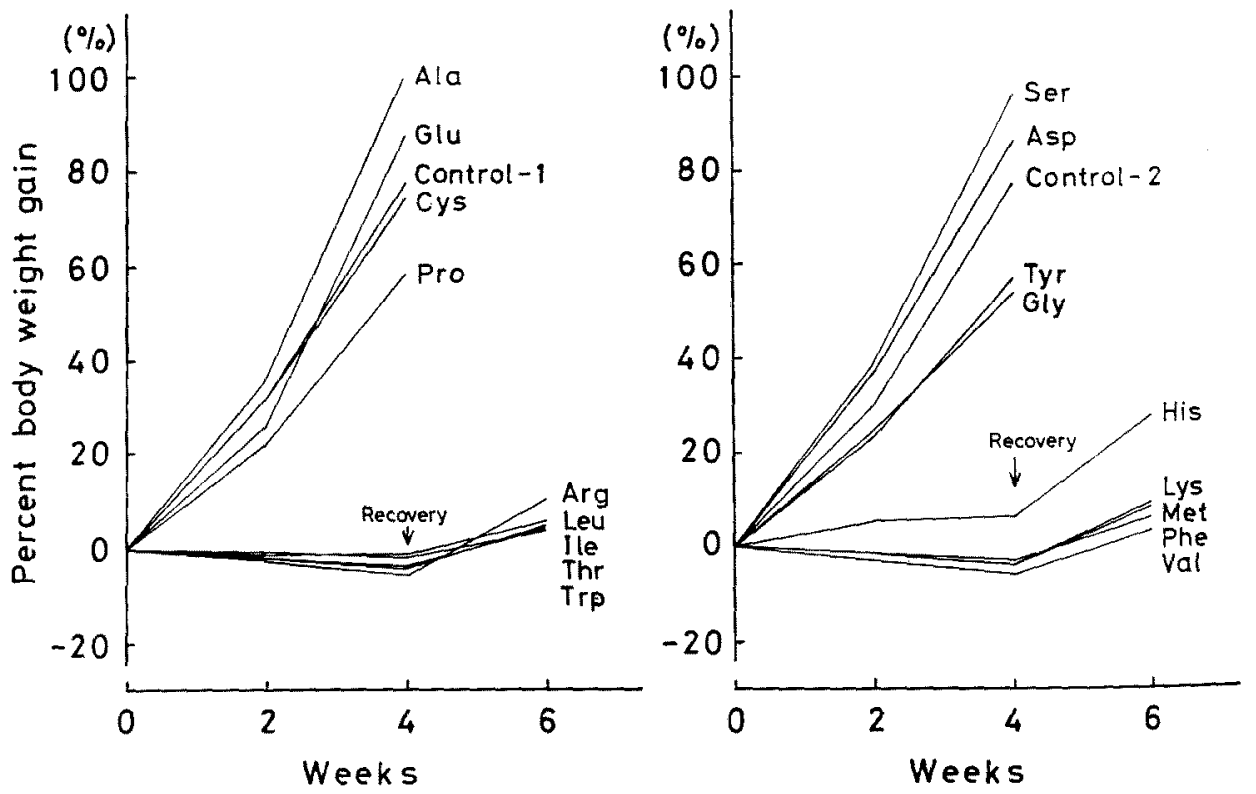

Fig. 3. Growth of experimental fish on amino acid-deficient diets. 
highest rate $(51 \%)$ of occurence was in isoleucine-deficient group followed by methionine-, leucine-, and tryptophan-deficient groups $(43,31$, and $26 \%$ ). There was no apparent alleviation of the deformity within the recovery test for 2 weeks. The symptom, however, appeared also in the control group, though only a few fish were affected. To ascertain whether the disease was epidemic or not, a similar experiment was carried out twice. In these experiments, the congestion was not recognized, but lordosis appeared similarly, and the deformity appeared much more frequently in essential amino acid-deficient groups. A few fish in both nonessential amino acid-deficient and complete diet groups, however, showed the same symptom.

\section{Discussion}

In the present study, carp were found to grow to some extent on an amino acid test diet neutralized with $\mathrm{NaOH}$, but only slightly on the diet not neutralized. Carp is stomachless, and digestion is considered to proceed on neutral or slightly alkaline side in the digestive tract. If the amino acid diet which is too acidic is fed, the intestinal content of carp is supposed to be kept on acidic side in a considerable period of digestion due to the strong buffer action of amino acid mixture, and this seems to disturb the assimilation of amino acids.

The growth of young carp on the amino acid test diet was fairly inferior to that on the comparable casein diet, but seemed to be enough to examine the essential amino acids by the ommission test. It was thus postulated that carp are not different from other fish, so far as the kinds of essential amino acid are concerned. The reason why AOE et al. ${ }^{6 /}$ failed to sustain the growth of carp with the neutralized amino acid test diet is still obscure. It seems to be partly attributable to their low feeding rate. They gave carp the diet equivalent to $3 \%$ of the body weight daily by feeding four times a day. Since we fed carp ad libitum six times a day, the food intake in a 4 -week period is estimated to be nearly 3 to 4 times as much as that in the experiment of AOE et al. It is most likely that the amino acid test diet could not sustain growth at a low feeding rate due to its low feed efficiency.

A very low feed efficiency of amino acid test diet in carp is an interesting problem. In our experiment, carp increased their body weight more than twice within 6 weeks but the feed efficiency ranged usually between 15 to $20 \%$ on dry basis and the growth did not exceeded about $60 \%$ of that on the comparable casein diet under the same conditions. It is well established that salmonids show no difference in growth between the casein-gelatin diet and the corresponding amino acid test diet. This may suggest that carp have some peculiarity in protein nutrition.

Salmonids, catfish and eel have been reported to show retarded appetite shorty after being fed amino acid test diet deficient in an essential amino acid. In the case of carp, depression of appetite was not so clear and the fish fed actively the essential amino acid- 
deficient diet even at the end of 4 weeks.

The congestion observed in this experiment was epidemic, while the cause of the other symptom, lordosis, was not decided. Halver et al. observed lordosis in tryptophan deficient sockeye salmon ${ }^{21}$ and rainbow trout ${ }^{3 \prime}$ as an essential amino acid-deficiency symptom. In carp, however, the symptom appeared also in nonessential amino acid-deficient and control groups, even though in a very low frequency. Further experiment should be necessary to decide the etiological factor.

\section{References}

1) J. E. Halver, D. C. Delong, and E. T. Mertz: J. Nutrition, 63, 95-105 (1957).

2) J. E. Halver and W. E. Shanks: ibid., 72, 340-346 (1960).

3) W. E. Shanks, G. D. Gahimer, and J. E. Halver: Prog. Fish-Cult., 24, 68-73 (1962).

4) H. K. Dupree and J. E. HAlver: Trans. Amer. Fish. Soc., 99, 90-92 (1970).

5) S. ARai, T. Nose, and Y. Hashimoto: This Bull., 38, 753-759 (1972).

6) H. Aoe, I. Masuda, I. Abe, T. Saito, T. Toyoda, and S. Kitamura: ibid., 36, 407-413 (1970). 